\title{
Funciones del psicólogo en las organizaciones ¿Soy indispensable?
}

\author{
Willian Sierra-Barón \\ Magíster en Educación \\ Universidad Surcolombiana, Neiva, Colombia \\ Correo electrónico: willian.sierra@gmail.com
}

Luz Adriana Hernández Perdomo

Psicóloga

Universidad Surcolombiana, Neiva, Colombia Correo electrónico: adrianahernandez517@gmail.com

Kelly Yohana Medina Narváez

Psicóloga

Universidad Surcolombiana, Neiva, Colombia Correo electrónico: kellyyohana9304@outlook.com

Andrés Gómez Acosta

Magíster en Psicología

Universidad de Pamplona

Correo electrónico: cesar.gomez@unipamplona.edu.co

Recibido: $16 / 04 / 2020$

Evaluado: 09/06/2020

Aceptado: 04/08/2020

\section{Resumen}

La investigación tuvo como objetivo conocer desde la perspectiva de algunos actores organizacionales, la importancia que tienen los profesionales en Psicología en la realización de sus funciones laborales organizacionales y, a su vez, identificar que otros profesionales pueden desarrollar estas mismas funciones. La metodología se orientó por un estudio descriptivo ex post facto con diseño de solo post test. La recolección de información se realizó a través de un cuestionario ad hoc diseñado para los propósitos de la investigación. Participaron 106 actores organizacionales de cuatro municipios del sur de Colombia. El estudio concluye, desde la perspectiva de los participantes, que la mayoría de las funciones deben ser ejercidas por psicólogos, aunque se resalta que algunas pueden ser realizadas por Administradores de Empresas, profesionales en Salud y Seguridad en el Trabajo, Ingenieros Industriales y Civiles.

Palabras clave

Organización, psicólogo, empleador, funciones, profesional.

8 Para citar este artículo: Sierra, W., Hernández, L.A., Medina, K.Y. y Gómez-Acosta, A. (2022). Funciones del psicólogo en las organizaciones ¿Soy indispensable?. Informes Psicológicos, 22(1), pp. 131-150 http://dx.doi.org/10.18566/infpsic.v22n1a08 


\title{
The duties of a psychologist in organizations: Am I indispensable?
}

\section{Abstract}

The objective of the research was to know, from the perspective of some organizational actors, the importance of professionals in Psychology in the performance of their organizational work duties and to identify what other professionals can develop these same functions. The methodology was guided by an ex post facto descriptive study with a single post-test design. The information collection was carried out through an ad hoc questionnaire designed for the purposes of the research. 106 organizational actors from four municipalities in southern Colombia participated. The study concludes, from the perspective of the participants, that most of the duties should be performed by psychologists, although it is highlighted that some can be performed by Business Administrators, professionals in Health and Safety at Work, Industrial and Civil Engineers.

\section{Funções do psicólogo nas organizações, sou indispensável?}

\begin{abstract}
Resumo
0 objetivo da pesquisa foi conhecer, na perspectiva de alguns atores organizacionais, a importância dos profissionais da Psicologia no desempenho de suas funções organizacionais de trabalho e, por sua vez, identificar que outros profissionais podem desenvolver essas mesmas funções. A metodologia foi orientada por um estudo descritivo ex post facto com um desenho apenas pós-teste. A coleta de informações foi realizada por meio de um questionário ad hoc elaborado para os fins da pesquisa. Participaram 106 atores organizacionais de quatro municípios do sul da Colômbia. 0 estudo conclui, na perspetiva dos participantes, que grande parte das funções deve ser desempenhada por psicólogos, embora se destaque que algumas podem ser desempenhadas por Administradores de Empresas, profissionais da Saúde e Segurança do Trabalho, Engenheiros Industriais e Civis.
\end{abstract}

Palavras chave Organização, psicólogo, empregador, funções, profissional. 


\section{ntroducción}

A través de la historia, la sociedad se ha ido sumergiendo en procesos de globalización y modernización, que incluyen el desarrollo de las organizaciones, generando cambios sobre aspectos ambientales, políticos y económicos a partir de su responsabilidad social y compromiso con el entorno (Segredo, 2013).

Las organizaciones se asumen como un sistema de elementos interrelacionados, basados en una estructura social con una tendencia evolutiva al cambio permanente, producto de una causalidad circular y multifactorial, compuesta por individuos en constante interacción con recursos tecnológicos, financieros y humanos, cuyo fin es alcanzar objetivos específicos (Bonilla, 1998; Daft, 2000). En Colombia la Ley 905 de 2004 promueve el desarrollo de micro, pequeñas y medianas empresas, con el fin de estimular, favorecer y constituir organizaciones competitivas.

Así, las organizaciones al cumplir un papel esencial dentro de la sociedad deben promover procesos que faciliten el crecimiento empresarial que incluya el fomento del bienestar y calidad de vida laboral en su interior y, a su vez, aumente su competitividad y productividad (Camargo, 2013; Gómez, 2016). Dichos procesos de crecimiento contrastan con la dinámica propuesta desde la perspectiva del desarrollo organizacional, la cual está caracterizada por la orientación del empresario en direccionar la organización y determinar las necesidades para el bienestar laboral del talento humano, implementando estrategias donde la calidad, efectividad y competitividad, se logren hacia el mejoramiento continuo de sus prácticas organizacionales (Caicedo \& Acosta, 2015; Enciso \& Perilla, 2004).

Para alcanzar el direccionamiento esperado, el empresario es consciente de la necesidad en la organización de un profesional cualificado que le oriente el fortalecimiento de la competitividad, reconociendo y empoderando las fortalezas de su recurso humano (Caicedo \& Acosta, 2015). Por esto, resulta indispensable reconocer la importancia del rol del psicólogo en las organizaciones (Álvarez, Estrella \& Rosas, 2018), quienes realizan programas, proyectos y acciones hacia el mejoramiento del bienestar del recurso humano mejorando la calidad de vida relacionada con el trabajo y promoviendo el cumplimiento de la normatividad (Mejía, Bravo \& Montoya, 2012; Sierra-Barón, Pineda, Rodríguez \& Matta-Santofimio, 2020).

Así, la Psicología Organizacional, como especialidad o campo aplicado de la psicología, está orientada al estudio y aplicación de principios científicos en el contexto del trabajo (Spector, 2002); no obstante es importante enmarcar que para algunos autores, la Psicología en este campo aplicado históricamente da cuenta de dos divisiones: de una parte, la industrial, con una mayor orientación a la eficiencia organizacional en procesos como diseño de cargos, selección, capacitación y evaluación del personal; por otra parte, la división organizacional, cuyo foco se enmarca más hacia el interés en el empleado y su comportamiento, así como 
el fortalecimiento de su bienestar en el lugar de trabajo (Enciso \& Perilla, 2004; Morocho, 2002). Otros autores describen este campo, en términos de Psicología Organizacional y del Trabajo, para referirse "al conocimiento multidisciplinar del comportamiento de individuos y grupos situados en el campo de la estructura y funcionamiento de las organizaciones" (Malvezzi, 2016, p.28).

El psicólogo en el área empresarial se desempeña en distintos escenarios de las organizaciones y del trabajo, diseña, lidera e implementa proyectos y programas de gerencia y gestión humana fomentando el logro de las metas organizacionales y sociales hacia el incremento del bienestar psicológico y la calidad de vida de las personas (Colegio Colombiano de Psicólogos, 2013). En Colombia, con la entrada en vigencia de la Ley 1090 (Congreso de Colombia, 2006), se reglamenta el ejercicio de la profesión y se dicta el código deontológico y bioético para el ejercicio de la Psicología. En las organizaciones, el Psicólogo debe contar con habilidades, aptitudes y conocimientos que le permitan desarrollar mecanismos de acción, trabajo grupales e individuales, enfatizados en los nuevos retos organizacionales que el mundo global ofrece (Enciso \& Peridilla, 2004; González, Rueda \& Santana, 2017). No obstante, lo que la experiencia muestra es que otros profesionales (ejemplo: trabajador social), también desempeñan funciones de gestión del talento humano $u$ otros procesos asociados (Chaparro \& Urra, 2014).

Sin embargo, en algunos países latinoamericanos las demandas del rol del psicólogo y sus funciones tienen mayor relevancia en las áreas clínica y educativa, en tanto que el área organizacional puede llegar a tener menos demanda por parte de los empleadores y más cuando se trata de pequeñas y medianas empresas (Bravo, Baquero \& Valadez, 2012; Martínez \& Galindo, 2012; Pavón, 2017).

Los empleadores requieren que el Psicólogo desempeñe tareas como reclutamiento y selección de personal, labores administrativas de capacitación y docencia; esto enmarcado en actividades de evaluación, diagnóstico, planeación, intervención e investigación. Esto se puede contrastar con lo dispuesto por la Sociedad de Psicología Industria y Organizacional, SIOP por sus siglas en inglés (división 14 de la Asociación Americana de Psicología, 2020), quienes describen este campo de la Psicología como aquel que estudia el lugar de trabajo, a partir del uso de sus conocimientos y métodos científicos sobre aspectos de gran importancia para los negocios, incluyendo la gestión del talento humano, la evaluación, la selección, la capacitación, el desarrollo organizacional, la motivación, el liderazgo y el desempeño. No obstante, las funciones del psicólogo se encuentran desvinculadas de muchos procesos organizacionales y en algunos casos su labor no genera un valor agregado, por lo que resulta relevante promover el rol del psicólogo en la gestión humana dentro de las organizaciones (Cabrera, Hickman \& Mares, 2010; López, Molina \& Sánchez, 2014; Ortega, 2006; Pantoja, 2017; Rivera, 2004; Sierra-Barón et al., 2020), así como en todos los procesos que propendan por la calidad de vida laboral y el desarrollo organizacional. 
Las funciones asociadas a la gestión del talento humano son desempeñadas mayormente por profesionales no psicólogos, evidenciando la poca asociación entre el rol del psicólogo en las organizaciones y los intereses de los empleadores para su vinculación. Gerentes, subgerentes, administradores de empresas, comunicadores sociales, ingenieros industriales, economistas, abogados y antropólogos, son quienes mayormente desempeñan funciones asociadas a la gestión del talento humano (Hernández, Hernández \& Maldonado, 2016; Jaramillo, 2005; Suárez, 2014; Urbina, 2011). Al respecto, algunos autores han planteado cómo la gestión del talento humano puede implicar para una mejor comprensión y análisis, una perspectiva multidisciplinaria e incluso compleja (Del Rio Cortina \& Velásquez, 2015; García, 2019; Larrea, 2019; Márquez, 2014; Rivero \& Díaz, 2008).

Desde un juicio ético en todas las organizaciones el área de gestión del talento humano, por ser la responsable del desempeño de sus colaboradores, debe estar orientada por el psicólogo facultado en conocimientos para la oportuna intervención de riesgos psicosociales que se presentan en la organización (Suárez, 2014), así como todas aquellas que se deriven de su ejercicio hacia la promoción y cuidado de la salud mental de los miembros que conforman la organización.

A este respecto Sierra-Barón et al. (2020) encontraron en un estudio cuya finalidad fue describir las competencias, funciones y ámbitos de actuación de psicólogos que laboran en el contexto organizacional, una amplia gama de acciones y procesos que los profesionales en psicología reconocen que realizan al interior de las organizaciones. Los participantes de este estudio refirieron ser más competentes en conocimientos, actitudes, valores éticos, desarrollo profesional y en conocer, argumentar y aplicar conceptos de psicología organizacional; las funciones más desempeñadas por los psicólogos son las de elaborar y presentar informes de manera oral y escrita, promover el clima organizacional, trabajar en equipos interdisciplinarios y capacitar al personal. Reubicar a los empleados dentro de la organización, apoyar la definición de políticas salariales, el reconocimiento de méritos, y realizar procesos de investigación se encuentran entre las funciones de menor desempeño.

Así las cosas y reconociendo la importancia de aportes científicos que dan cuenta del rol del profesional en psicología desde la perspectiva del psicólogo, resulta pertinente y relevante generar evidencia científica que explore desde la perspectiva de otros actores organizacionales (gerentes, coordinadores y líderes de procesos, empleadores, etc.), que permita contribuir al contraste de las perspectivas, para, de esta forma, continuar contribuyendo en el análisis del rol del psicólogo en las organizaciones.

En adición a lo anterior, resulta prioritario conocer si en las organizaciones existen los profesionales cualificados encargados del cambio y mantenimiento de estrategias, estructuras y sistemas las cuales garanticen el bienestar del recurso humano (Perea, 2010), es decir, que fomenten la gestión de cambio, entendida como el proceso a través del cual las organizaciones se transforman 
desde su estado actual o inicial, a un futuro deseado para aumentar su efectividad, encontrando mejores formas de utilizar sus recursos y capacidades para crear valor y aumentar su desempeño (Jones, 2008).

Además, conocer desde la perspectiva de algunos actores organizacionales, la importancia de que Psicólogos realicen funciones dentro de las organizaciones y, a su vez, identificar que otros profesionales pueden desarrollar estas mismas funciones. En este sentido, las preguntas que orientaron la presente investigación fueron: desde la perspectiva de algunos actores organizacionales ¿Qué importancia tiene que el profesional en psicología realice funciones dentro de las organizaciones? ¿Qué otros profesionales pueden desarrollar las funciones que realizan los psicólogos?

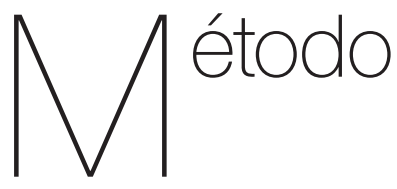

Esta investigación corresponde a un estudio descriptivo ex post facto con diseño de solo post test (Montero \& León, 2007).

\section{Participantes}

Participaron de manera voluntaria 106 actores organizacionales (46.2\% hombres y $53.8 \%$ mujeres), vinculados laboralmente a empresas de los municipios de Neiva (55.7\%), Gigante (24.5\%),
Garzón (15.1\%) y Altamira (4.7 \%) del departamento del Huila, con cargos administrativos y directivos (50.9\%), asesor, auxiliar, operario (38.7\%) y psicólogo (cargo con esta denominación), líder del Sistema de Salud y Seguridad en el Trabajo y líder de Recursos Humanos (10.4\%).

Las empresas a las que están vinculados los participantes de la investigación tienen una antigüedad de 1 a 2 años (20.8\%), 3 a 5 años (15.1\%), 6 y 10 años (23.6\%) y 10 o más años (40.6\%); su tipo de capital es privado (95.3\%), público (2.8\%) y mixto (1.9\%); su clasificación según la actividad es primario $(0.9 \%)$, secundario $(21.7 \%)$, terciario (74.5\%) y cuaternario (2.8\%). El sector de la economía corresponde a comercial (60.4\%), servicios (34.9\%) e industrial $(4.7 \%)$ y la clasificación según su tamaño y número de empleados es microempresa (39.6\%), pequeña empresa (29.2\%), mediana empresa (18.9\%) y macro empresa (12.3\%).

\section{Instrumentos}

Para la recolección de información se creó un cuestionario ad hoc para consultar la importancia de la realización de las funciones del psicólogo en las organizaciones. El instrumento consta de dos apartados: el primero incluye información relacionada con características de la organización y si labora o no un psicólogo; la segunda, se basa en la Ficha de identificación de funciones del Psicólogo de las Organizaciones y del Trabajo, elaborada por Pineda \& Rodríguez (2016), que indaga acerca de la importancia y necesidad del desarrollo de las funciones 
del Psicólogo en las organizaciones. E coeficiente alfa de Cronbach para estos ítems fue .88. Este segundo apartado incluye 39 ítems y se subdividen, a su vez, en dos apartados: el primero estipula una valoración de 0 a 4 donde 0 representa la valoración más baja y 4 la más alta, para cada una de las funciones; en su segunda parte abre la posibilidad al encuestado de responder si la función que realiza el Psicólogo en las organizaciones la puede realizar otro profesional (incluyendo una respuesta dicótoma Si/No), para posteriormente indicar qué profesional podría realizar la mencionada función. El coeficiente alfa de Cronbach para los 39 ítems fue .92.

Al finalizar la encuesta se indaga acerca de la importancia de que el psicólogo realice asesoría en 17 posibles áreas de la organización, incluyendo una valoración de 0 a 4, donde 0 representa la valoración más baja y 4 la más alta para cada una de las áreas.

\section{Procedimiento}

Para el proceso de recolección de la información, se contactaron formalmente 200 empresas, distribuidas en los municipios de Altamira, Garzón, Gigante y Neiva, del departamento del Huila, de las cuales decidieron participar voluntariamente un total de 106 personas. Se logró acceder a éstas a través de encuentros en los que se dio a conocer el proyecto de investigación incluyendo sus implicaciones éticas y deontológicas. El proceso de recolección de información se adelantó entre los meses de agosto y diciembre de 2018.
La investigación se desarrolló en el marco de las orientaciones de la Ley 1090 de 2006, por la cual se reglamenta el ejercicio de la profesión de Psicología y se dicta el código deontológico y bioético. Para el análisis de datos se usó el programa IBM SPSS $®$ versión 23.

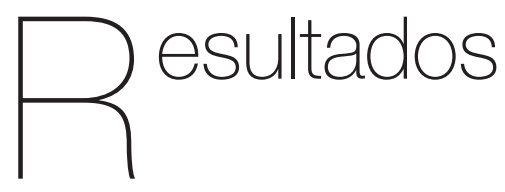

Los resultados muestran que solo en el $30.2 \%$ de las organizaciones participantes labora un Psicólogo, presentando las siguientes características laborales: un $64.3 \%$ tienen un contrato a término indefinido, el $32.1 \%$ tiene contrato a término fijo y el $3.6 \%$ restante tienen contrato por prestación de servicios; en cuanto a la antigüedad laboral del psicólogo dentro de la empresa se encontró que el 30.4\% tienen una antigüedad laboral entre 1 y 2 años, el $43.5 \%$ entre 3 a 4 años, el $8.7 \%$ entre 5 a 7 años, y el 17.4\% restante de 8 años o más; en cuanto al salario se halló que el 93\% de los psicólogos tienen una remuneración económica entre 1 a 4 salarios mínimos legales vigentes (smlv) y el $7 \%$ de los psicólogos restantes de 5 o más smlv.

La Tabla 1 muestra la valoración que los participantes dan a la importancia de que psicólogos realicen funciones dentro de las organizaciones. Para efectos de la presente investigación, en las tablas 1 y 2 las puntuaciones cercanas a 4.0 son consideradas altas y las más cercanas a 1 son consideradas bajas. 
Tabla 1.

Valoración de participantes sobre la importancia que psicólogos realicen funciones dentro de las organizaciones.

\begin{tabular}{|c|c|c|c|c|}
\hline Funciones & M & DS & Min & Max \\
\hline 1. Analizar los sistemas de compensación & 3.01 & .687 & 1 & 4 \\
\hline 2. Analizar y describir puestos de trabajo. & 3.14 & .821 & 1 & 4 \\
\hline $\begin{array}{l}\text { 3. Aportar en la conformación de trabajos enfocados a las metas y objetivos de } \\
\text { la organización. }\end{array}$ & 3.30 & .654 & 2 & 4 \\
\hline 4. Aportar en los procesos de la comunicación organizacional. & 3.41 & 678 & 1 & 4 \\
\hline 5. Apoyar el seguimiento de la cultura organizacional. & 3.38 & .598 & 2 & 4 \\
\hline 6. Apoyar la creación del departamento de gestión humana. & 3.46 & .644 & 2 & 4 \\
\hline 7. Apoyar la definición de políticas salariales y el reconocimiento de méritos. & 3.20 & .800 & 1 & 4 \\
\hline 8. Apoyar la desvinculación voluntaria e involuntaria de la organización. & $\underline{2.96}$ & .972 & 1 & 4 \\
\hline 9. Apoyar la realización de eventos de integración en la organización. & 3.44 & .775 & 0 & 4 \\
\hline 10. Apoyar los procesos de gestión de calidad. & 3.38 & .753 & 0 & 4 \\
\hline 11. Apoyar y realizar labores de bienestar laboral. & 3.42 & 678 & 2 & 4 \\
\hline 12. Apoyar y realizar proceso de reinducción. & 3.21 & .653 & 2 & 4 \\
\hline 13. Asesorar la gerencia. & $\underline{2.90}$ & .886 & 1 & 4 \\
\hline 14. Capacitar al personal. & 3.45 & 620 & 2 & 4 \\
\hline 15. Definir las políticas de gestión del talento humano. & 3.46 & 658 & 1 & 4 \\
\hline 16. Desarrollar competencias laborales. & 3.41 & .653 & 2 & 4 \\
\hline 17. Desarrollar planes de carrera. & 3.30 & .745 & 1 & 4 \\
\hline 18. Desarrollar y realizar seguimiento de programas de capacitación de personal. & 3.50 & .624 & 2 & 4 \\
\hline 19. Diagnosticar Clima Organizacional. & 3.54 & .557 & 2 & 4 \\
\hline 20. Diagnosticar necesidades de capacitación y desarrollo de personal. & 3.48 & .657 & 2 & 4 \\
\hline $\begin{array}{l}\text { 21. Elaborar perfiles psicológicos en diferentes ámbitos laborales a partir del } \\
\text { análisis de puestos y tareas. }\end{array}$ & 3.53 & .664 & 1 & 4 \\
\hline 22. Elaborar y presentar informes de manera oral y escrita. & $\underline{2.90}$ & 1.068 & 0 & 4 \\
\hline 23. Evaluar e intervenir la dinámica grupal en la organización. & 3.35 & .606 & 2 & 4 \\
\hline 24. Evaluar y diagnosticar e intervenir sobre conflictos. & 3.59 & .550 & 2 & 4 \\
\hline 25. Identificar e intervenir los factores de riesgo psicosociales. & 3.44 & .718 & 1 & 4 \\
\hline 26. Identificar y asesorar estilos de liderazgo. & 3.30 & .695 & 1 & 4 \\
\hline $\begin{array}{l}\text { 27. Liderar los procesos humanos mediante la gestión y desarrollo del talento } \\
\text { humano. }\end{array}$ & 3.54 & .558 & 2 & 4 \\
\hline 28. Potenciar la efectividad organizacional. & 3.44 & .555 & 2 & 4 \\
\hline $\begin{array}{l}\text { 29. Prestar asistencia emocional en accidentes de trabajo a nivel preventivo y } \\
\text { curativo. }\end{array}$ & 3.49 & .651 & 2 & 4 \\
\hline 30. Promover acciones con apoyo de asesores externos. & $\underline{2.93}$ & .930 & 1 & 4 \\
\hline 31. Promover el clima organizacional. & 3.42 & .678 & 1 & 4 \\
\hline 32. Realizar evaluación de desempeño. & 3.43 & .695 & 0 & 4 \\
\hline 33. Realizar inducciones. & 3.45 & .682 & 2 & 4 \\
\hline Funciones & M & DS & Min & Max \\
\hline
\end{tabular}


Continuación

\begin{tabular}{|c|c|c|c|c|}
\hline $\begin{array}{l}\text { 34. Realizar procesos de reclutamiento y selección del personal según el perfil } \\
\text { requerido. }\end{array}$ & 3.55 & .638 & 2 & 4 \\
\hline 35. Realizar seguimiento al logro de objetivos. & 3.40 & .633 & 2 & 4 \\
\hline 36. Realizar o apoyar procesos de investigación. & 3.16 & .719 & 1 & 4 \\
\hline 37. Retroalimentar a la totalidad de la organización. & 3.09 & .763 & 1 & 4 \\
\hline 38. Reubicar a los empleados dentro de la organización. & 2.99 & .888 & 1 & 4 \\
\hline 39. Trabajar en equipos interdisciplinarios. & 3.00 & .834 & 1 & 4 \\
\hline
\end{tabular}

Nota: Las funciones con puntuaciones más altas están en negritas. Las funciones con puntuaciones más bajas están resaltadas en negro y subrayadas. M = media; DS = Desviación estándar; Min = Puntuación de valoración mínima de cada función; Max = Puntuación de valoración máxima de cada función.

Según la valoración de los participantes sobre la importancia desarrollo de las funciones por profesionales en Psicología en las empresas, los ítems con tendencia alta fueron $18,19,21,27$, y 34 , relacionados con funciones como desarrollar y realizar seguimiento de programas de capacitación del personal, elaborar perfiles psicológicos en diferentes ámbitos laborales a partir del análisis de puestos y tareas, liderar los procesos humanos mediante la gestión y desarrollo del talento humano, realizar procesos de reclutamiento y selección del personal según el perfil requerido, siendo las funciones más valoradas ya que los empleadores las consideran necesarias e importantes en el desarrollo adecuado de la organización.
De igual forma, los ítems con tendencia baja fueron 8, 13, 22 y 30; lo que sugiere que funciones como apoyar la desvinculación voluntaria e involuntaria de la organización, asesorar la gerencia, elaborar y presentar informes de manera oral y escrita, y promover acciones con apoyo de asesores externos son las funciones menos valoradas y consideradas de menos necesidad de desarrollo dentro de la organización por los profesionales en psicología.

La Tabla 2 presenta el porcentaje según la valoración de los participantes frente a la pregunta de si las funciones pueden ser desarrolladas por otros profesionales diferentes al psicólogo.

Tabla 2.

Percepción de la importancia de que las funciones sean desarrolladas por otro profesional distinto al psicólogo (\%)

\begin{tabular}{|c|c|c|c|}
\hline Funciones & Sí\% & No\% & $\mathrm{N} / \mathrm{S} \%$ \\
\hline 1. Analizar los sistemas de compensación & 17.0 & 74.5 & 8.5 \\
\hline 2. Analizar y describir puestos de trabajo. & 20.8 & 75.5 & 3.8 \\
\hline $\begin{array}{l}\text { 3. Aportar en la conformación de trabajos enfocados a las metas } \\
\text { y objetivos de la organización. }\end{array}$ & 21.7 & 76.4 & 1.9 \\
\hline 4. Aportar en los procesos de la comunicación organizacional. & 20.8 & 76.4 & 2.8 \\
\hline 5. Apoyar el seguimiento de la cultura organizacional. & 17.0 & 79.2 & 3.8 \\
\hline 6. Apoyar la creación del departamento de gestión humana. & 14.2 & 79.2 & 6.6 \\
\hline $\begin{array}{l}\text { 7. Apoyar la definición de políticas salariales y el reconocimiento } \\
\text { de méritos. }\end{array}$ & 19.8 & 76.4 & 3.8 \\
\hline $\begin{array}{l}\text { 8. Apoyar la desvinculación voluntaria e involuntaria de la } \\
\text { organización. }\end{array}$ & 15.1 & 79.2 & 5.7 \\
\hline $\begin{array}{l}\text { 9. Apoyar la realización de eventos de integración en la } \\
\text { organización. }\end{array}$ & 23.6 & 75.5 & .9 \\
\hline
\end{tabular}




\begin{tabular}{|c|c|c|c|}
\hline Funciones & Sí\% & No\% & $\mathrm{N} / \mathrm{S} \%$ \\
\hline 10. Apoyar los procesos de gestión de calidad. & 25.5 & 72.6 & 1.9 \\
\hline 11. Apoyar y realizar labores de bienestar laboral. & 21.7 & 77.4 & 0.9 \\
\hline 12. Apoyar y realizar proceso de reinducción. & 19.8 & 75.5 & 4.7 \\
\hline 13. Asesorar la gerencia. & 21.7 & 72.6 & 5.7 \\
\hline 14. Capacitar al personal. & 26.4 & 72.6 & .9 \\
\hline 15. Definir las políticas de gestión del talento humano. & 15.1 & 79.2 & 5.7 \\
\hline 16. Desarrollar competencias laborales. & 13.2 & 81.1 & 5.7 \\
\hline 17. Desarrollar planes de carrera. & 10.4 & 72.6 & 17.0 \\
\hline $\begin{array}{l}\text { 18. Desarrollar y realizar seguimiento de programas de capaci- } \\
\text { tación de personal. }\end{array}$ & 17.0 & 80.2 & 2.8 \\
\hline 19. Diagnosticar Clima Organizacional. & 9.4 & 86.8 & 3.8 \\
\hline $\begin{array}{l}\text { 20. Diagnosticar necesidades de capacitación y desarrollo de } \\
\text { personal. }\end{array}$ & 16.0 & 79.2 & 4.7 \\
\hline $\begin{array}{l}\text { 21. Elaborar perfiles psicológicos en diferentes ámbitos labora- } \\
\text { les a partir del análisis de puestos y tareas. }\end{array}$ & 2.8 & 87.7 & 9.4 \\
\hline 22. Elaborar y presentar informes de manera oral y escrita. & 20.8 & 73.6 & 5.7 \\
\hline 23. Evaluar e intervenir la dinámica grupal en la organización. & 15.1 & 82.1 & 2.8 \\
\hline 24. Evaluar, diagnosticar e intervenir sobre conflictos. & 13.2 & 84.0 & 2.8 \\
\hline 25. Identificar e intervenir los factores de riesgo psicosociales. & 12.3 & 87.7 & 0 \\
\hline 26. Identificar y asesorar estilos de liderazgo. & 11.3 & 81.1 & 7.5 \\
\hline $\begin{array}{l}\text { 27. Liderar los procesos humanos mediante la gestión y desarro- } \\
\text { Ilo del talento humano. }\end{array}$ & 12.3 & 82.1 & 5.7 \\
\hline 28. Potenciar la efectividad organizacional. & 19.8 & 78.3 & 1.9 \\
\hline $\begin{array}{l}\text { 29. Prestar asistencia emocional en accidentes de trabajo a nivel } \\
\text { preventivo y curativo. }\end{array}$ & 15.1 & 84.9 & 0 \\
\hline 30. Promover acciones con apoyo de asesores externos. & 24.5 & 70.8 & 4.7 \\
\hline 31. Promover el clima organizacional. & 11.3 & 86.8 & 1.9 \\
\hline 32. Realizar evaluación de desempeño. & 15.1 & 82.1 & 2.8 \\
\hline 33. Realizar inducciones. & 20.8 & 76.4 & 2.8 \\
\hline $\begin{array}{l}\text { 34. Realizar procesos de reclutamiento y selección del personal } \\
\text { según el perfil requerido. }\end{array}$ & 11.3 & 85.8 & 2.8 \\
\hline 35. Realizar seguimiento al logro de objetivos. & 22.6 & 74.5 & 2.8 \\
\hline 36. Realizar o apoyar procesos de investigación. & 15.1 & 71.7 & 13.2 \\
\hline 37. Retroalimentar a la totalidad de la organización. & 20.8 & 74.5 & 4.7 \\
\hline 38. Reubicar a los empleados dentro de la organización. & 20.8 & 69.8 & 9.4 \\
\hline 39. Trabajar en equipos interdisciplinarios. & 13.2 & 74.5 & 12.3 \\
\hline
\end{tabular}

Nota: Si = La función puede ser realizada por cualquier otro profesional diferente a un Psicólogo; № = La función debe ser realizada únicamente por un profesional en psicología; Ns = El encuestado no sabe no responde.

Según la valoración de los participantes frente a la pregunta de si las funciones pueden ser ejercidas por otro profesional no psicólogo, la respuesta con tendencia alta es No, ubicándose en un rango de $69.8 \%-87.7 \%$ del total de las 39 
funciones, lo que sugiere que, en general, según la percepción de los participantes, la mayoría de las funciones no pueden ser realizadas por otro profesional distinto al psicólogo. De igual forma, se encontró una tendencia baja a la respuesta $\mathrm{Si}$, ubicándose en un rango de 2.8\%-26.4\% del total de las 39 funciones. Dentro de las respuestas $\mathrm{Si}$, las funciones que puntúan tendencia alta fueron 9, 10, 14 y 30, advirtiendo que funciones como apoyar la realización de eventos de integración en la organización, apoyar los procesos de gestión de calidad, capacitar al personal y promover acciones con apoyo de asesores externos, son las funciones que pueden ser realizadas por otro profesional de manera eficiente.

La Tabla 3 muestra los porcentajes de las valoraciones acerca de los profesionales que los participantes consideran cualificados para desarrollar las funciones del psicólogo en la organización. Para efectos de la presente investigación, en las tablas 3 y 4 los porcentajes cercanos a 100 son considerados altos y los más cercanos a 1 son consideradas bajos.

Tabla 3.

Profesionales considerados cualificados para ejercer las funciones del psicólogo en la organización

\begin{tabular}{|c|c|c|c|c|c|c|}
\hline Funciones & $\begin{array}{l}\text { Admón. de } \\
\text { Empresas \% }\end{array}$ & SST \% & $\begin{array}{l}\text { Ing. Ind. } \\
\text { y civil \% }\end{array}$ & Otros \% & Psicólogo \% & Total \% \\
\hline 1. Analizar los sistemas de compensación. & 9.43 & 3.77 & 2.8 & 0 & $\underline{84.0}$ & 100 \\
\hline 2. Analizar y describir puestos de trabajo. & 8.49 & 4.72 & 7.6 & 0 & $\underline{79.3}$ & 100 \\
\hline $\begin{array}{l}\text { 3. Aportar en la conformación de traba- } \\
\text { jos enfocados a las metas y objetivos de la } \\
\text { organización. }\end{array}$ & 13.21 & 4.72 & 3.8 & 1.89 & $\underline{76.4}$ & 100 \\
\hline $\begin{array}{l}\text { 4. Aportar en los procesos de la comunicación } \\
\text { organizacional. }\end{array}$ & 14.15 & 3.77 & 2.8 & 2.83 & $\underline{76.4}$ & 100 \\
\hline $\begin{array}{l}\text { 5. Apoyar el seguimiento de la cultura } \\
\text { organizacional. }\end{array}$ & 8.49 & 3.77 & 4.7 & 3.77 & $\underline{79.3}$ & 100 \\
\hline $\begin{array}{l}\text { 6. Apoyar la creación del departamento de } \\
\text { gestión humana. }\end{array}$ & 5.66 & 2.83 & 5.7 & 6.6 & $\underline{79.3}$ & 100 \\
\hline $\begin{array}{l}\text { 7. Apoyar la definición de políticas salariales y } \\
\text { el reconocimiento de méritos. }\end{array}$ & 10.38 & 3.77 & 5.7 & 3.77 & $\underline{76.4}$ & 100 \\
\hline $\begin{array}{l}\text { 8. Apoyar la desvinculación voluntaria e invo- } \\
\text { luntaria de la organización. }\end{array}$ & 7.55 & .94 & 5.7 & 6.6 & $\underline{79.3}$ & 100 \\
\hline $\begin{array}{l}\text { 9. Apoyar la realización de eventos de integra- } \\
\text { ción en la organización. }\end{array}$ & 14.15 & 3.77 & 6.6 & .94 & $\underline{74.5}$ & 100 \\
\hline 10. Apoyar los procesos de gestión de calidad. & 11.32 & 6.6 & 7.6 & 1.89 & $\underline{72.6}$ & 100 \\
\hline $\begin{array}{l}\text { 11. Apoyar y realizar labores de bienestar } \\
\text { laboral. }\end{array}$ & 10.38 & 7.55 & 3.8 & .94 & $\underline{77.4}$ & 100 \\
\hline 12. Apoyar y realizar proceso de reinducción. & 9.43 & 5.66 & 4.7 & 5.66 & $\underline{74.5}$ & 100 \\
\hline 13. Asesorar la gerencia. & 10.38 & .94 & 10.4 & 5.66 & $\underline{72.6}$ & 100 \\
\hline 14. Capacitar al personal. & 14.15 & 9.43 & 2.8 & 1.89 & $\underline{71.7}$ & 100 \\
\hline $\begin{array}{l}\text { 15. Definir las políticas de gestión del talento } \\
\text { humano. }\end{array}$ & 7.55 & .94 & 6.6 & 6.6 & $\underline{78.3}$ & 100 \\
\hline 16. Desarrollar competencias laborales. & 11.32 & 0 & 2.8 & 6.6 & $\underline{79.3}$ & 100 \\
\hline
\end{tabular}




\begin{tabular}{|c|c|c|c|c|c|c|}
\hline Funciones & $\begin{array}{l}\text { Admón. de } \\
\text { Empresas \% }\end{array}$ & SST $\%$ & $\begin{array}{l}\text { Ing. Ind. } \\
\text { y civil \% }\end{array}$ & Otros $\%$ & Psicólogo \% & Total \% \\
\hline 17. Desarrollar planes de carrera. & 7.55 & 0 & 3.8 & 9.43 & $\underline{79.3}$ & 100 \\
\hline $\begin{array}{l}\text { 18. Desarrollar y realizar seguimiento de pro- } \\
\text { gramas de capacitación de personal. }\end{array}$ & 9.43 & 2.83 & 5.7 & 2.83 & $\underline{79.3}$ & 100 \\
\hline 19. Diagnosticar Clima Organizacional. & 4.72 & 2.83 & 2.8 & 3.77 & $\underline{85.9}$ & 100 \\
\hline $\begin{array}{l}\text { 20. Diagnosticar necesidades de capacitación } \\
\text { y desarrollo de personal. }\end{array}$ & 6.6 & 3.77 & 6.6 & 4.72 & $\underline{78.3}$ & 100 \\
\hline $\begin{array}{l}\text { 21. Elaborar perfiles psicológicos en diferen- } \\
\text { tes ámbitos laborales a partir del análisis de } \\
\text { puestos y tareas. }\end{array}$ & 1.89 & 0 & .9 & 9.43 & $\underline{87.7}$ & 100 \\
\hline $\begin{array}{l}\text { 22. Elaborar y presentar informes de manera } \\
\text { oral y escrita. }\end{array}$ & 10.38 & 3.77 & 6.6 & 5.66 & $\underline{73.6}$ & 100 \\
\hline $\begin{array}{l}\text { 23. Evaluar e intervenir la dinámica grupal en } \\
\text { la organización. }\end{array}$ & 9.43 & 1.89 & 3.8 & 4.72 & $\underline{80.2}$ & 100 \\
\hline $\begin{array}{l}\text { 24. Evaluar y diagnosticar e intervenir sobre } \\
\text { conflictos. }\end{array}$ & 6.6 & 3.77 & 2.8 & 4.72 & $\underline{82.1}$ & 100 \\
\hline $\begin{array}{l}\text { 25. Identificar e intervenir los factores de ries- } \\
\text { go psicosociales. }\end{array}$ & .94 & 7.55 & 3.8 & 0 & $\underline{87.7}$ & 100 \\
\hline 26. Identificar y asesorar estilos de liderazgo. & 7.55 & .94 & 2.8 & 9.43 & $\underline{79.3}$ & 100 \\
\hline $\begin{array}{l}\text { 27. Liderar los procesos humanos mediante la } \\
\text { gestión y desarrollo del talento humano. }\end{array}$ & 2.83 & 3.77 & 5.7 & 7.55 & $\underline{80.2}$ & 100 \\
\hline 28. Potenciar la efectividad organizacional. & 14.15 & .94 & 4.7 & 3.77 & $\underline{76.4}$ & 100 \\
\hline $\begin{array}{l}\text { 29. Prestar asistencia emocional en acciden- } \\
\text { tes de trabajo a nivel preventivo y curativo. }\end{array}$ & 5.66 & 7.55 & 1.9 & 1.89 & $\underline{83.0}$ & 100 \\
\hline $\begin{array}{l}\text { 30. Promover acciones con apoyo de asesores } \\
\text { externos. }\end{array}$ & 10.38 & 8.49 & 4.7 & 5.66 & $\underline{70.8}$ & 100 \\
\hline 31. Promover el clima organizacional. & 8.49 & .94 & 1.9 & 3.77 & $\underline{84.9}$ & 100 \\
\hline 32. Realizar evaluación de desempeño. & 5.66 & 4.72 & 4.7 & 4.72 & $\underline{80.2}$ & 100 \\
\hline 33. Realizar inducciones. & 10.38 & 5.66 & 4.7 & 4.72 & $\underline{74.5}$ & 100 \\
\hline $\begin{array}{l}\text { 34. Realizar procesos de reclutamiento y se- } \\
\text { lección del personal según el perfil requerido. }\end{array}$ & 4.72 & 2.83 & 2.8 & 4.72 & $\underline{84.9}$ & 100 \\
\hline 35. Realizar seguimiento al logro de objetivos. & 13.21 & 4.72 & 4.7 & 4.72 & $\underline{72.6}$ & 100 \\
\hline $\begin{array}{l}\text { 36. Realizar o apoyar procesos de } \\
\text { investigación. }\end{array}$ & 9.43 & 2.83 & 3.8 & 15.09 & $\underline{68.9}$ & 100 \\
\hline $\begin{array}{l}\text { 37. Retroalimentar a la totalidad de la } \\
\text { organización. }\end{array}$ & 16.04 & .94 & 2.8 & 4.72 & $\underline{75.5}$ & 100 \\
\hline $\begin{array}{l}\text { 38. Reubicar a los empleados dentro de la } \\
\text { organización. }\end{array}$ & 16.04 & .94 & 3.8 & 9.43 & $\underline{69.8}$ & 100 \\
\hline 39. Trabajar en equipos interdisciplinarios. & 6.6 & 1.89 & 4.7 & 14.15 & $\underline{72.6}$ & 100 \\
\hline
\end{tabular}

Nota: Las valoraciones están dadas en porcentajes, subrayadas se encuentran los porcentajes de los psicólogos para cada una de las funciones y en negrita las valoraciones mal altas de los distintos profesionales, en las diferentes funciones.

Según la valoración de los participantes frente a la respuesta dicótoma SI, los profesionales que consideran cualificados para ejercer las funciones del psicólogo son los Administradores de Empresas, los profesionales Salud y Seguridad en el Trabajo (SST), los Ingenieros Industriales y Civiles. 
De acuerdo a los resultados, los Administradores de Empresas se consideran mejor cualificados para realizar las funciones del psicólogo tales como: aportar en la conformación de trabajos enfocados a las metas y objetivos de la organización, aportar en los procesos de la comunicación organizacional, apoyar la realización de eventos de integración en la organización, capacitar al personal, potenciar la efectividad organizacional, realizar seguimiento al logro de objetivos, retroalimentar a la totalidad de la organización, y reubicar a los empleados dentro de la organización.

Los profesionales en SST son considerados mejor cualificados para remplazar al psicólogo en el desarrollo de funciones como: apoyar y realizar labores de bienestar laboral, apoyar y realizar proceso de reinducción, identificar e intervenir los factores de riesgo psicosociales, prestar asistencia emocional en accidentes de trabajo a nivel preventivo y curativo y promover acciones con apoyo de asesores externos.

Por último, los Ingenieros Industrial y Civil son considerados más cualificados para remplazar al psicólogo en el desarrollo de funciones como: apoyar los procesos de gestión de calidad, analizar y describir puestos de trabajo, y asesorar la gerencia.

Finalmente, la Tabla 4 muestra los porcentajes de la valoración de los participantes frente a la necesidad e importancia de que los profesionales en psicología asesoren y participen en el desarrollo de los procesos de las áreas: Salud y Seguridad en el Trabajo, Gestión del Talento Humano, Contabilidad, Finanzas, Marketing, Asesoría Jurídica, Administrativa, Producción, Soporte técnico, Comercial, Comunicación, Técnica, Tesorería, Innovación y Tecnología, Investigación, Gerencia, Planeación.

Tabla 4.

Valoración de las asesorías del profesional en psicología a las áreas de la organización (\%)

\begin{tabular}{|c|c|c|c|c|c|c|c|}
\hline Área & 0 & 1 & 2 & 3 & 4 & No Aplica & Total \\
\hline $\begin{array}{l}\text { Salud y seguridad } \\
\text { en el trabajo }\end{array}$ & $5.7 \%$ & $17.9 \%$ & $20.8 \%$ & $14.2 \%$ & $13.2 \%$ & $28.3 \%$ & $100 \%$ \\
\hline Gestión del talento humano & $0 \%$ & $1.9 \%$ & $2.8 \%$ & $39.6 \%$ & $42.5 \%$ & $13.2 \%$ & $100 \%$ \\
\hline Contabilidad & $7.5 \%$ & $24.5 \%$ & $10.4 \%$ & $15.1 \%$ & $34.9 \%$ & $7.5 \%$ & $100 \%$ \\
\hline Finanzas & $5.7 \%$ & $17.9 \%$ & $20.8 \%$ & $14.2 \%$ & $13.2 \%$ & $28.3 \%$ & $100 \%$ \\
\hline Marketing & $2.8 \%$ & $\underline{21.7 \%}$ & $15.1 \%$ & $17.9 \%$ & $20.8 \%$ & $21.7 \%$ & $100 \%$ \\
\hline Asesoría Jurídica & $7.5 \%$ & $20.8 \%$ & $17.9 \%$ & $11.3 \%$ & $18.9 \%$ & $23.6 \%$ & $100 \%$ \\
\hline Administrativa & $1.9 \%$ & $13.2 \%$ & $16.0 \%$ & $27.4 \%$ & $32.1 \%$ & $9.4 \%$ & $100 \%$ \\
\hline Producción & $4.7 \%$ & $14.2 \%$ & $17.0 \%$ & $18.9 \%$ & $14.2 \%$ & $31.1 \%$ & $100 \%$ \\
\hline Soporte técnico & $9.4 \%$ & $20.8 \%$ & $13.2 \%$ & $12.3 \%$ & $12.3 \%$ & $32.1 \%$ & $100 \%$ \\
\hline Comercial & $2.8 \%$ & $11.3 \%$ & $15.1 \%$ & $28.3 \%$ & $32.1 \%$ & $10.4 \%$ & $100 \%$ \\
\hline Comunicación & $1.9 \%$ & $8.5 \%$ & $17.9 \%$ & $18.9 \%$ & $31.1 \%$ & $21.7 \%$ & $100 \%$ \\
\hline Técnica & $9.4 \%$ & $14.2 \%$ & $17.9 \%$ & $11.3 \%$ & $8.5 \%$ & $38.7 \%$ & $100 \%$ \\
\hline
\end{tabular}




\begin{tabular}{|c|c|c|c|c|c|c|c|}
\hline Área & 0 & 1 & 2 & 3 & 4 & No Aplica & Total \\
\hline Tesorería & $11.3 \%$ & $\underline{15.1 \%}$ & $18.9 \%$ & $8.5 \%$ & $12.3 \%$ & $34.0 \%$ & $100 \%$ \\
\hline Innovación y tecnología & $2.8 \%$ & $17.0 \%$ & $17.9 \%$ & $14.2 \%$ & $17.0 \%$ & $31.1 \%$ & $100 \%$ \\
\hline Investigación & $0 \%$ & $6.6 \%$ & $13.2 \%$ & $25.5 \%$ & $23.6 \%$ & $31.1 \%$ & $100 \%$ \\
\hline Gerencia & $0 \%$ & $4.7 \%$ & $18.9 \%$ & $26.4 \%$ & $34.9 \%$ & $15.1 \%$ & $100 \%$ \\
\hline Planeación & $.9 \%$ & $5.7 \%$ & $18.9 \%$ & $24.5 \%$ & $21.7 \%$ & $28.3 \%$ & $100 \%$ \\
\hline
\end{tabular}

Nota: en esta tabla están contenidas cada una de las áreas de la organización y su valoración en porcentajes de 1 a 4.Siendo 1 la valoración mínima y 4 la valoración máxima; están subrayadas las áreas con mayor puntuación en los rangos de valoraciones mínimas y resaltadas en negro las valoraciones en los rangos de mayores.

De acuerdo con la Tabla 4, las áreas más valoradas por los participantes frente a la necesidad e importancia de que los psicólogos asesoren y participen en el desarrollo de las diferentes áreas de la organización son: Gestión del Talento Humano, Contabilidad, Administrativa, Comercial, Comunicación, Investigación, Gerencia, y Planeación; en contraste, las áreas con menor valoración son: Marketing, asesoría jurídica y tesorería.

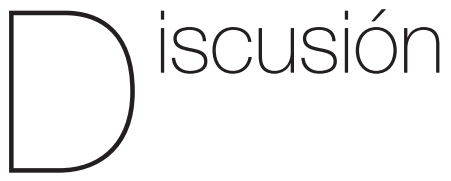

El objetivo de este estudio fue conocer, desde la perspectiva de algunos actores organizacionales, la importancia de que los psicólogos realicen funciones dentro de las organizaciones y, a su vez, identificar qué otros profesionales pueden desarrollar estas mismas funciones.

Los resultados de esta investigación sugieren, desde la perspectiva de los participantes, que las funciones del psicólogo en las organizaciones tiene poca representación, la cual se evidencia en su ausencia en micro y pequeñas empresas; esto podría afectar de manera directa el avance efectivo de las personas en las organizaciones, dado que el rol del psicólogo es fundamental en el desarrollo del recurso humano, mediante el acompañamiento de las personas desde el ámbito organizacional, estableciendo un equilibrio en el crecimiento de éstas y de la organización, con una visión renovadora de cambio generando mayor productividad y desarrollo empresarial (Cárdenas, 2013; Santana, 2014).

En este sentido, la valoración dada por los participantes frente a la importancia del desarrollo de las funciones por parte del profesional en psicología sugiere que entre las funciones más valoradas están desarrollar y realizar seguimiento de programas de capacitación del personal, elaborar perfiles psicológicos en diferentes ámbitos laborales a partir del análisis de puestos y tareas, liderar los procesos humanos mediante la gestión y desarrollo del talento humano, realizar procesos de reclutamiento y selección del personal según el perfil requerido. Esto se puede contrastar con los hallazgos del estudio de Borja y Pérez (2005) quienes refieren, en el campo organizacional, que el psicólogo debe centrarse en funciones de procesos de capacitación, reclutamiento, selección de personal, evaluación del desempeño, análisis del puesto y de funciones; igualmente, estos hallazgos 
pueden compararse con los de Santana (2014) y Foster (2011), cuando afirman que las funciones del Psicólogo deben ir encaminadas a evaluar, promover, fomentar y mejorar los procedimientos que se llevan a cabo en la organización para generar estrategias que aumenten la satisfacción de los empleados en relación con su trabajo y con la organización. A su vez, estos resultados no contrastan con los hallazgos de Sierra-Barón et al. (2020) quienes, frente al rol del Psicólogo Organizacional (desde la perspectiva de los psicólogos), hallaron que las funciones más desempeñadas son las de elaborar y presentar informes de manera oral y escrita, promover el clima organizacional, trabajar en equipos interdisciplinarios y capacitar al personal. Esto sugiere que puede haber diferencias entre las perspectivas de los psicólogos y otros actores organizacionales acerca de las funciones.

Por otro lado, los resultados muestran que la mayoría de las funciones deben ser ejercidas estrictamente por psicólogos, ya que estos poseen competencias y habilidades específicas que permiten el correcto desenvolvimiento mediante conocimiento de teorías, métodos de evaluación, diagnóstico, promoción, prevención, tratamiento e investigación; aunque también señalan que en algunas funciones específicas los profesionales en psicología pueden ser sustituidos por profesionales formados en competencias generales; en este sentido, se evidenció que, dentro de las funciones que pueden ser ejercidas por profesionales distintos al psicólogo, los empleadores consideran cualificados a: los administradores de empresas, los profesionales en SG-SST, los ingenieros industriales y los ingenieros civiles, entre otros; esto concuerda con lo planteado por Urbina (2011), al referir que disciplinas como Administración de Empresas, Ingeniería Industrial, y profesionales en Salud y Seguridad en el Trabajo han incursionado en el desarrollo de los procesos de Gestión del Talento Humano en las organizaciones.

Además, el presente estudio evidenció que los participantes destacan que las áreas en las que el Psicólogo presenta más apoyo son gestión del talento humano, contabilidad, administrativa, comercial, comunicación, investigación, gerencia, y planeación. Esto se puede contrastar con hallazgos como los de Muñoz (2015), cuando plantea que el Psicólogo debe ofrecer soporte a todas las áreas de la organización, estableciendo estrategias basadas en el análisis organizacional de alta dirección mediante asesorías, liderando equipos para lograr un desarrollo y rendimiento ascendente dentro de las organizaciones, generando así mayor productividad empresarial (Borja \& Pérez, 2005). Dicho establecimiento de estrategías orientado en el marco de una linea de acción desde la gestión estratégica de recursos humanos, implica comprender la artículación entre los procesos individuales y organizacionales (Storey, Ulrich \& Wrigth, 2019).

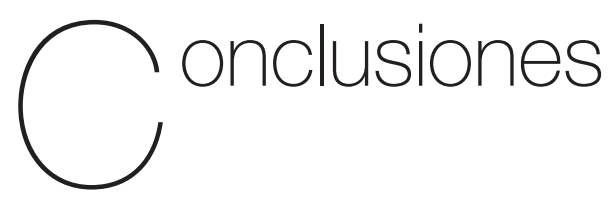

Es importante evidenciar que la valoración acerca de la importancia de las funciones que los psicólogos realizan dentro de las organizaciones es poco representativa en micro y pequeñas empresas, lo cual no quiere decir que en dichas entidades no se considere necesario e importante el acompañamiento de estos profesionales para el desarrollo de distintos procesos. 
Las funciones con mayor valoración por parte de los empleadores son desarrollar y realizar seguimiento de programas de capacitación del personal, elaborar perfiles psicológicos en diferentes ámbitos laborales a partir del análisis de puestos y tareas, liderar los procesos humanos mediante la gestión y desarrollo del talento humano, realizar procesos de reclutamiento y selección del personal según el perfil requerido; así mismo, se encontró que las funciones menos valoradas son: apoyar la desvinculación voluntaria e involuntaria de la organización, asesorar la gerencia, elaborar y presentar informes de manera oral y escrita, y promover acciones con apoyo de asesores externos.

Desde la perspectiva de los participantes, la mayoría de las funciones deben ser ejercidas por los profesionales en psicología, aunque es importante resaltar que algunas funciones específicas pueden ser desarrolladas de manera eficiente por otros profesionales como los administradores de empresas, los profesionales en SST, Ingenieros Industriales y Civiles.

Finalmente, las áreas de la organización donde más representación tiene el Psicólogo son gestión del talento humano, contabilidad, administrativa, comercial, comunicación, investigación, gerencia, y planeación; de igual forma, las áreas de menos participación del psicólogo son Marketing, asesoría jurídica y tesorería.

En el grupo de actores organizacionales consultados, los resultados de esta investigación sugieren una importancia relativa de los profesionales en Psicología en la realización de sus funciones laborales organizacionales, así como la compresión y consideración de otros profesionales en el desempeño de estas funciones, lo que podría explicarse por la multidisciplinariedad que implica el abordaje de estos procesos en los contextos organizacionales.

\section{Limitaciones}

Durante el desarrollo de la investigación surgieron situaciones que dificultaron algunos procesos para la recolección de la información. Una de éstas correspondió a la falta de tiempo disponible por parte de potenciales participantes, por lo que la investigación no contó con una muestra significativa; además, la falta de conexión e interacción del sector productivo y la universidad influyó negativamente, ya que se hizo difícil la interacción efectiva con las organizaciones; otra limitación fue la extensión del instrumento de recolección de información, que pudo eventualmente inducir fatiga en algunos de los encuestados.

Se recomienda para futuras investigaciones continuar con el mejoramiento psicométrico del cuestionario y ampliar la cantidad de municipios y departamentos para que la muestra de participantes de las empresas se incremente con el fin de lograr que la valoración tenga mayor alcance, así como incluir grandes empresas en el proceso de recolección de información.

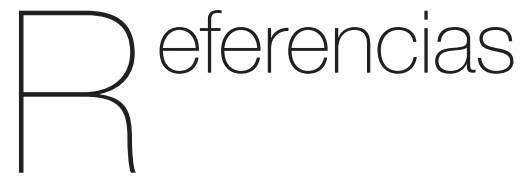

Álvarez, L. A., Estrella, B. M., \& Rosas, S. M. (2018). Rol del Psicólogo Organizacional en la gestión de Talento Humano. PODIUM, (33), 79-90. doi: 10.31095/ podium.2018.33.8 
Bonilla, J. (1998). Laempresayelempresario. Revista de Derecho Privado, 3, 105126. Recuperado de https://heinonline. org/HOL/LandingPage?handle=hein. journals/revdpriv3\&div=9\&id=\&page=

Borja, I., \& Pérez, E. (2005). Rol y posicionamiento del psicólogo organizacional en las empresas de la ciudad de Cartagena de Indias. (Tesis de Licenciatura). Universidad Tecnológica de Bolívar, Cartagena, Colombia.

Bravo, M., Vaquero, J., \& Valadez, A. (2012). Las competencias profesionales percibidas por estudiantes de psicología. Revista de Educación y Desarrollo, 21, 13-20. Recuperado de http://www.cucs.udg.mx/revistas/edu_ desarrollo/anteriores/21/021_Bravo.pdf

Cabrera, R., Hickman, H., \& Mares, G. (2010). Perfil profesional del psicólogo requerido por empleadores en entidades federativas con diferente nivel socioeconómico en México. Revista Enseñanza e Investigación en Psicología, 15(2), 257-271. Recuperado de https://www.redalyc.org/ pdf/292/29215980002.pdf

Caicedo, V., \& Acosta, A. (2015). La gestión del talento humano y el ser humano como sujeto de desarrollo. Revista Politécnica, 8(14), 105-113. Recuperado de https://revistas.elpoli.edu.co/index. php/pol/article/view/216

Camargo, F. C. (2013). Reformas al mercado laboral para estimular la productividad, competitividad y calidad de vida en el Estado de México. Revista Cofactor, 4(7), 7-94. Recuperado de https://dialnet.unirioja.es/servlet/ articulo?codigo $=4839582$
Cárdenas, F. (2013). El rol del psicólogo organizacional en un mercado globalizado. (Proyecto de Grado). Universidad ICESI, Cali, Colombia.

Chaparro, Y. C., \& Urra, M. (2014). Competencias específicas del trabajador social en la gestión del talento humano. Revista Tendencias \& Retos, 19(2), 27-44. Recuperado de https://dialnet.unirioja.es/servlet/ articulo?codigo $=4924414$

Colegio Colombiano de Psicólogos (2013). Perfiles por competencias profesional en psicología del deporte, jurídico, neurociencias y psicobiología, organizacional y psicología social: ambiental, comunitaria y política. Recuperado de http://www. colpsic. org.co/imagenes/Perfiles_por_ competencia_del_profesional_en_ psicologia.pdf.

Congreso de Colombia (6 de septiembre de 2006). Ley 1090. DO: 46.383. Recuperada de http://www. secretariasenado.gov.co/senado/ basedoc/ley_1090_2006.html

Daft, R. (2000). Teoríaydiseñoorganizacional (10ed, Cap. 1). México: International Thomson. Recuperado de https:// s3.amazonaws.com/academia.edu. documents/51891185/LIBRO_DISENO_ ORGANIZACIONAL.pdf?AWSAccessK eyld=AKIAIWOWYYGZ2Y53UL3A\&Exp ires $=1534358106 \&$ Signature $=q 1 \mathrm{mRQ7}$ kqcEwubs2E6pmc8Z6Ej88\%3D\&respo nse-content-disposition=inline $\% 3 \mathrm{~B} \% 20$ filename\%3DTeoria_y_diseno_ organizacional.pdf

Del Rio Cortina, A. \& Velásquez, J.R. (2015). Propuesta de análisis del talento humano 
a partir del pensamiento complejo. Revista Logos, Ciencia \& Tecnología, 7(1), 35-46. Recuperado de https://www. redalyc.org/pdf/5177/517751487004.pdf

Enciso, E. \& Perilla, L. (2004). Visión retrospectiva, actual y prospectiva de la psicología organizacional. Acta Colombiana de Psicología, (11), 5-22. Recuperado de https:// actacolombianapsicologia.ucatolica. edu.co/article/view/476

Foster, L. (2011). Industrialand Organizational Psychology in Latin America: The Peruvian Story. Society for industrial and Organizational Psychology, 4(48). Recuperado de http://www.siop.org/tip/ april11/11thompson.aspx

García, Y. (2019). Conjugación del talento humano y la gestión pública de lo político, lo público, lo institucional, Io estratégico. Revista Interdisciplinaria de Humanidades, Educación, Ciencia y Tecnología, 5(8), 174- 189. Recuperado de https://dialnet.unirioja.es/servlet/ articulo?codigo $=7088736$

Gómez, M. (2016). Sobre la psicología organizacional y del trabajo en Colombia. Revista Colombiana de Ciencias Sociales, 7(1), 131-153. Recuperado de https://dialnet.unirioja. es/servlet/articulo?codigo $=5454161$

González, L., Rueda, L., \& Santana, G. (2017). Proyectos de intervención y gestión humana en sitios de práctica organizacional desarrollados en el marco del programa de psicología de la Universidad Piloto de Colombia. Revista Enfoques, 2(1), 119-135. http://dx.doi. org/10.24267/23898798.212
Hernández, A., Hernández, X., Maldonado, J. (2016). Estudio correlacional entre las competencias del psicólogo organizacional en relación con otras carreras, de acuerdo a las necesidades del análisis de puestos de recursos humanos en Cancún. Centro de Investigaciones Turísticas. Cancún, México. Recuperado de http://lasallecancun.edu.mx/old/ wp-content/uploads/2016/07/ PsicologiaArticulo012016.pdf

Jaramillo, O. L. (2005). Gestión del Talento Humano en la Micro, Pequeña y Mediana empresa vinculada al programa Expopyme de la Universidad del Norte en los sectores de confecciones y alemientos. Revista Pensamiento y Gestión, 18, 103-137. Recuperado de https://www.redalyc. org/pdf/646/64601805.pdf

Jones, G. (2008). Teoría Organizacional, Diseño y Cambio en las Organizaciones. 5ta Edición. México: Pearson Education.

Larrea, M.D. (2019). Sistematización de la experiencia de trabajo interdisciplinario desde la perspectiva de $\mathrm{RRHH}$ para el proyecto Zoom 2019 (Tesis de Licenciatura). Universidad Casa Grande. Recuperado de http://dspace. casagrande.edu.ec:8080/handle/ ucasagrande/2028

Lopez, A., Molina, C., \& Sánchez, K. (2014). El rol del psicólogo laboral, sus funciones y aportes a las organizaciones donde se han desempeñado como gerentes de recursos humanos (Tesis Doctoral). Universidad de El Salvador, San Salvador, El Salvador. 
Malvezzi, S. (2016). Psicologíaorganizacional y del trabajo. De la administración científica a la globalización: una historia de desafíos. En J. Orejuela, V. Andrade \& M. Villamizar. Psicología de las organizaciones y del trabajo. Apuestas de investigación II. Colombia: Editorial Bonaventuriana.

Márquez, J.L. (2014). Desarrollo de las organizaciones complejas bajo una perspectiva sinérgica del talento humano. Visión general, 2, 273-290. Recuperado de https://www.redalyc. org/pdf/4655/465545897003.pdf

Martínez, L., \& Galindo, R. (2012). Caracterización del roly posicionamiento actual de los psicólogos de gestión humana en cuatro empresas de Bogotá (Trabajo de grado). Universidad Santo Tomás. Bogotá, Colombia.

Mejía, A., Bravo, M., \& Montoya, A. (2012). El factor del talento humano en las organizaciones: análisis desde una perspectiva crítica/humana. Revista Ingeniería Industrial, 4, 2-11. Recuperado de http://scielo.sld.cu/pdf/ rii/v34n1/rii02113.pdf

Montero, I., \& León, O. (2007). A guide for naming research studies in Psychology. International Journal of Clinical \& Health Psychology, 7, 847-862. Recuperado de https://www.redalyc. org/pdf/337/33770318.pdf

Morocho, L. (2008). La Psicología Organizacional y los retos del nuevo milenio. Revista Liberabit, (8), 63-69. Recuperado de http://revistaliberabit. com/en/resultado_en.php
Muñoz, P. (2015). Perfil de competencias del Psicólogo Organizacional para las empresas privadas del servicio de Quito (Tesis de Licenciatura). Universidad de las Américas, Quito, Ecuador.

Ortega, M. (2006). Ausencia de un departamento de recursos humanos en la organización. (Trabajo de grado). Universidad de La Sabana, Chía, Colombia.

Pantoja, D. L. (2017). La psicología organizacional y los procesos de Gestíon de Talento. Boletín Informativo CEl, 4(2), 77-80. Recuperado de http:// editorial.umariana.edu.co/revistas/ index.php/BoletinlnformativoCEl/article/ view/1376

Pavón, F.E. (2017). Análisis comparativo del rol del psicólogo, en las áreas educativa, empresarial y clínica; en la última década, en la ciudad de Ibarra (Trabajo de Grado). Universidad Técnica del Norte, Ecuador. Recuperado de http://repositorio.utn.edu.ec/ handle/123456789/7213

Perea, R. (2010). Propuesta de estrategia para la evaluación del desempeño laboral de los médicos en Cuba. Revista cubana de Educación Médica Superior, 24(3), 387-417. Recuperado de http:// scielo.sld.cu/pdf/ems/v24n3/ems11310. pdf

Pineda, J., \& Rodríguez, A. (2016). Competencias profesionales, amibtos de actuación y funciones del psicologo de las organizaciones y del trabajo en la ciudad de Neiva. (Tesis de Grado) Universidad Surcolombiana, Neiva, Colombia. 
Rivera, M. (2004). Representaciones sociales sobre psicólogos organizacionales (Trabajo de Grado). Universidad de La Sabana, Chía, Colombia.

Rivero, S., \& Díaz, M. (2008). La interdisciplinariedad en la organización de los procesos institucionales. Acimed, 18(6), 1-17. Recuperado de http://scielo. sld.cu/pdf/aci/v18n6/aci081208.pdf

Santana, R. (2014). El papel del psicólogo en la implantación de un sistema de seguridad en una empresa de trasporte local y foráneo de carga general en la ciudad de Toluca. (Memoria de práctica profesional). Universidad Autónoma del Estado de México, Toluca, México. Recuperado de http://ri.uaemex.mx/ handle/20.500.11799/41147

Segredo, A. (2013). Clima organizacional en la gestión del cambio para el desarrollo de la organización. Revista Cubana de Salud Pública, 39, 385-393. Recuperado de http://scielo.sld.cu/pdf/ rcsp/v39n2/spu17213.pdf

Sierra-Barón, W., Pineda, J., Rodríguez, A.M. \& Matta-Santofimio, J.D. (2020). Ejercicio profesional del psicólogo en el contexto del trabajo y las organizaciones. Informes Psicológicos, 20(1), 111-129. http://dx.doi.org/10.18566/infpsic. v20n1a08

Society for Industrial and Organizational Psychology - SIOP (2020). For Organizations $\mathrm{I}-\mathrm{O}$ and Employee Lifecycle. Recuperado de https://www. siop.org/Professionals

Spector, P. (2002). Psicología industrial y organizacional. Investigación y práctica. México: Manual Moderno.

Storey, J., Ulrich, D., \& Wright, P. M. (2019). Strategic Human Resource Management: A Research Overview. Routledge.

Suárez, N. (2014). Concepciones existentes sobre el trabajador, sus usos e implicaciones cognitivas en el quehacer del psicólogo organizacional. (Tesis doctoral). Universidad de Antioquia. Medellín, Colombia.

Urbina, C. (2011). El rol del Psicólogo en las Organizaciones Salvadoreñas Publicas y Privadas. (Tesis doctoral). Universidad Dr. José Matías Delgado, San Salvador, El Salvador. 